compression of the right main bronchus and the right pulmonary arterial wall focal calcification. She had not had any surgical intervention for AORPA and her symptoms got worse overtime.

Results Both, follow up CT chest and bronchoscopy revealed mechanical compression of the right main bronchus and extensive mucous plugging. CT chest [figure 1] demonstrated interval progression with the development of a new large area of consolidation in right middle and lower lobes, right perihilar bronchiectasis and reactive mediastinal lymph nodes. CMR confirmed the anomalous origin of the right pulmonary artery from the posterior aspect of the ascending aorta and displayed the features of right lung hyperaemia [figure 1]. The right pulmonary artery, aortic root and the ascending aorta were dilated.

Conclusion Anomalous right pulmonary artery is usually diagnosed in early childhood, but our patient was diagnosed in adult life possibly due to the early surgical treatment of PDA that might have delayed the clinical presentation. The delayed diagnosis increased her morbidity and affected the quality of life; therefore, we recommend early investigation and surgical management planning.

\section{AN AUDIT OF ADULT VTE RISK ASSESSMENT COMPLIANCE WITH NICE GUIDELINES}

${ }^{1}$ Sanjida Rahman, ${ }^{1}$ Sachin Ananth, ${ }^{1}$ Felicite Mukeshimana, ${ }^{2}$ Nicola Whiteley, ${ }^{2}$ Raymond Anakwe. ${ }^{1}$ Imperial College School of Medicine; ${ }^{2}$ Imperial College Healthcare NHS Trust

\subsection{6/postgradmedj-2018-fpm.33}

Introduction Venous Thromboembolisms (VTEs) are often preventable. In the UK, $60 \%$ of all VTEs are hospital-associated, costing the NHS approximately $£ 200$ million. VTE risk assessments ensure patient safety and reduce the economic healthcare burden of complications. According to NICE guidelines (NG89), risk assessments should be performed on all patients on admission, however, the NHS Quarter 4 2017/18 assessment showed 1992 inpatients were not VTE risk assessed at Imperial College NHS Trust (ICHT) between January to March 2018. This audit aimed to better understand the strengths and weaknesses of ICHT's performance to allow clinical leads and risk owners to formulate an action plan to close these gaps.
Subjects and methods The ICHT Adult VTE Trust policy was assessed using the relevant baseline assessment tool for NICE guidelines NG89. Seven quality statements corresponding to NICE Quality Standard 3 (QS3) and the Trust policy were used to develop the audit tool for assessing the Trust's performance. At St Mary's Hospital, 60 consecutive inpatients, across 6 different specialties, formed the sample population. Data was collected between 6th August until 13th August 2018 using ICHT electronic health record, CERNER. Analysis of the data applied ICHT Trust's Assurance rating for each sub-finding. Ethical principles and procedures adhered to standards set by the World Medical Association (WMA) Declaration of Helsinki and ICHT.

Results Eight of the total 60 patients, did not have accessible VTE risk assessment forms, either due to blank forms submitted $(2 / 8)$ or no form being submitted $(6 / 8)$. The mean time to initial assessment was 38.6 hours. There were four patients whose initial VTE assessments were documented more than 6 days after inpatient admission; 3 out of 4 of these exceeded 16 days post-admission. The audit Results showed that compliance rate of initial assessment and reassessment is currently below target at $91.7 \%$ and $78.2 \%$ respectively. Thirty-seven patients were at risk of thrombosis and had no contraindications to thromboembolism-deterrent stockings (TEDS). Despite this, 21 out of 37 of these patients were not prescribed TEDS. Forty two patients were prescribed pharmacological prophylaxis, however only 20 out of 42 had been weighed prior to prescription. A $0 \%$ compliance rate was found regarding documentation that high-risk patients received written VTE prevention information on admission.

Conclusions The main areas of improvement identified were to ensure VTE assessment and reassessment were completed for all inpatients and to reduce the mean time to initial assessment. Patient education should also be improved by providing written VTE prevention given to high-risk patients on admission and in pre-operative assessment, ensuring this is documented on CERNER. Mechanical prophylaxis (TEDs) should be appropriately prescribed to non-contraindicated patients at risk of thrombosis. In addition, the weighing of all patients prior to the prescription of pharmacological VTE prophylaxis also requires improvement to ensure the correct dose is given. A larger trust-wide audit is needed to consolidate these findings and follow patients to discharge to determine compliance to Statements 6 and 7 in NICE QS3. 